\title{
Tratamento farmacológico do transtorno obsessivo-compulsivo
}

\author{
Carla M arques
}

Unidade de Transtorno Obsessivo-Compulsivo (Programa de Ansiedade e Depressão) do Instituto de Psiquiatria da Universidade Federal do Rio de Janeiro. Rio de Janeiro, RJ, Brasil

\begin{abstract}
The pharmacotherapy for obsessive-compulsive disorder $(O C D)$ has started with clomipramine, a potent nonselective serotonin reuptake inhibitor. Newer drugs that selectively inhibit serotonin reuptake (SSRIs) such as fluvoxamine, fluoxetine, paroxetine, and sertraline are also effective and well-tolerated by OCD patients, even in high doses. Efficacy of venlafaxine and citalopram for OCD are under investigation. Randomized controlled trials of OCD pharmacotherapy, as well as positive results found in open trials and case reports are reviewed in this paper. Potent selective serotonin reuptake inhibitors are the pharmacotherapy of choice for OCD. If one SSRI is ineffective, others may be beneficial. OCD is frequently a chronic condition that requires long-term treatment.
\end{abstract}

Keywords Obsessive-compulsive disorder. Pharmacotherapy. Pharmacology. Clomipramine. Selective serotonin reuptake inhibitors.

\section{Introdução}

O transtorno obsessivo compulsivo (TOC) era considerado raro e de má resposta ao tratamento medicamentoso até menos de 20 anos. A utilização de antidepressivos que aumentam a transmissão serotoninérgica modificou esse quadro, e atualmente cerca de $60 \%$ dos pacientes apresentam melhora significativa, embora raramente fiquem assintomáticos.

Entre as hipóteses mais aventadas para a fisiopatologia do TOC, está a de que a neurotransmissão serotoninérgica está envolvida pelo menos na expressão dos sintomas. Esta se origina principalmente da observação de que antidepressivos que aumentam a função serotonérgica, como os inibidores de recaptação de serotonina (IRS) - sejam eles seletivos (fluoxetina, fluvoxamina, paroxetina, sertralina, citalopram) ou não (clomipramina) -, melhoram os sintomas obsessivo-compulsivos, enquanto os que agem preferencialmente sobre a noradrenalina (nortriptilina, desipramina) são pouco ou nada eficazes. Isto é diferente do que se costuma ver na depressão e no transtorno de pânico.

Neste artigo, abordar-se-ão questões referentes à psicofarmacoterapia clínica do TOC, como os medicamentos mais eficazes, as doses empregadas, as taxas de recaída e o tratamento de manutenção. Os IMAOs (inibidores da monoaminoxidase) serão discutidos no artigo de Shavitt et al, neste número, junto com o tratamento dos casos resistentes.

\section{Medicamentos eficazes \\ Clomipramina}

Logo após a introdução da clomipramina na Suíça em 1966, Fernandez \& Lopez Ibor ${ }^{1}$ descreveram melhora em 13 de 16 pacientes com "neurose obsessiva", tratados por 2 a 5 dias com clomipramina endovenosa. Desde então, inúmeros relatos de casos, estudos abertos e mais de dez estudos controlados com placebo demonstraram a eficácia da clomipramina no TOC. Ela passou a ser o padrão de comparação para estudos de eficácia de outros fármacos no TOC (padrão-ouro). O estudo mais importante sobre a eficácia da clomipramina no TOC envolveu 520 pacientes recrutados em 21 centros americanos. Dos 260 que usaram a clomipramina, cerca de $60 \%$ obtiveram uma redução média de $40 \%$ na sintomatologia obsessiva, comparados com cerca de $4 \%$ de resposta ao placebo. $^{2}$

\section{Inibidores seletivos de recaptação da serotonina (ISRS)}

Supondo-se que a resposta à clomipramina deva-se à potente inibição na recaptação de serotonina, os antidepressivos ISRS passaram a ser investigados no tratamento do TOC. Atualmente, há estudos multicêntricos envolvendo um grande número de pacientes demonstrando a eficácia da fluoxetina, ${ }^{3}$ da fluvoxamina, ${ }^{4}$ da sertralina ${ }^{5}$ e da paroxetina ${ }^{6}$. Estudos abertos utilizando o citalopram apontam também para uma possível eficácia no TOC. ${ }^{7}$

\section{Outros medicamentos}

A venlafaxina, um inibidor seletivo da recaptação de serotonina e noradrenalina, mostrou resultados favoráveis num estudo aberto ${ }^{8}$ e num duplo-cego com poucos pacientes. ${ }^{9} \mathrm{Ou}-$ tros medicamentos como neurolépticos, estabilizadores de humor e ansiolíticos, que são usados para casos refratários ou co-mórbidos, são discutidos no artigo de Shavitt et al.

\section{Manejo clínico \\ Uma vez que o TOC geralmente tem curso crônico, com os pacientes demorando em média sete anos para procurar ajuda, e que as opções de tratamento são limitadas, deve-se procurar iniciar com doses baixas da medicação e subir lentamente (cada 3 a 7 dias), conforme a tolerância do paciente. ${ }^{10}$ Isto diminui a}


incidência de efeitos colaterais e dá tempo para o surgimento de tolerância aos mesmos (Tabela).

\begin{tabular}{|c|c|c|c|}
\hline \multicolumn{4}{|c|}{$\begin{array}{l}\text { Tabela - Doses dos inibidores de recaptação de derotonina no tratamento } \\
\text { do TOC. }{ }^{11}\end{array}$} \\
\hline Medicação & $\begin{array}{l}\text { Dose } \\
\text { inicial }\end{array}$ & $\begin{array}{l}\text { Dose média } \\
\text { diária }\end{array}$ & $\begin{array}{l}\text { Faixa } \\
\text { terapêutica }\end{array}$ \\
\hline Clomipramina & 25 & 200 & $100-300$ \\
\hline Fluvoxamina & 50 & 200 & $100-300$ \\
\hline Fluoxetina & 20 & 50 & $20-80$ \\
\hline Sertralina & 50 & 150 & $75-225$ \\
\hline Paroxetina & 20 & 50 & $20-60$ \\
\hline
\end{tabular}

O tratamento pode ser iniciado com clomipramina ou com um ISRS, na dependência da história medicamentosa anterior e da existência de comorbidades clínicas ou psiquiátricas. Assim, devese evitar clomipramina num paciente com bloqueio atrioventricular ou risco de suicídio, preferir um ISRS quando houver comorbidade com fobia social e evitar benzodiazepínicos em pacientes com abuso de substâncias psicoativas ou personalidade borderline.

A orientação de utilizar os ISRS como primeira escolha ${ }^{11}$ no tratamento farmacológico do TOC vem sendo questionada por alguns autores. ${ }^{12}$ A clomipramina foi considerada mais eficaz que todos os ISRS em várias metanálises publicadas até o momento. ${ }^{13-19}$ Em que pesem as limitações desse tipo de estudo (amostras, critérios de inclusão e de resposta diferentes entre os ensaios revisados), chama a atenção que um percentual maior de pacientes tratados com a clomipramina sejam considerados "melhores" ou "muito melhores" em relação a pacientes tratados com fluoxetina, fluvoxamina ou sertralina. ${ }^{14} \mathrm{O}$ perfil de efeito colateral dos ISRS é mais benigno. Embora a clomipramina tenha uma taxa maior de efeitos colaterais, a interrupção do tratamento devido a eles não é maior que a dos ISRS. ${ }^{10}$ Este é um dado importante, já que o custo do tratamento com os ISRS tende a ser maior.

Embora vários estudos tenham utilizado doses altas de clomipramina (até $300 \mathrm{mg} / \mathrm{dia}$ ), doses superiores a $250 \mathrm{mg} / \mathrm{dia}$ devem ser evitadas, devido ao maior risco de convulsões tipo grande mal $\left(1,47 \%\right.$ ao ano $\left.^{2}\right)$.

A melhora nos sintomas geralmente custa a aparecer. Nos estudos multicêntricos, as diferenças entre medicamento e placebo só apareceram na segunda semana para a clomipramina e a sertralina, na quarta para a fluoxetina e, na sexta para a fluvoxamina. Não se pode dizer que um paciente não respondeu sem ter utilizado um IRS nas doses máximas toleradas por 10 a 12 semanas (fase aguda). Geralmente a resposta não é completa, mas se consegue uma redução média de $40 \%$ na intensidade dos sintomas em $40 \%$ a $60 \%$ dos casos. Alguns pacientes continuam a melhorar nos meses subseqüentes com a mesma medicação utilizada na fase aguda. Os que não responderam a um IRS podem responder a um segundo ou terceiro. ${ }^{20}$

\section{Doses}

Estudos utilizando diferentes doses de fluoxetina ${ }^{3}$ e de sertralina ${ }^{5}$ observaram uma curva dose-resposta plana, isto é: o aumento das doses não se acompanhava de aumento da resposta. No entanto, a experiência clínica mostra que vários pacientes que não responderam a baixas doses do fármaco melho- ram com o aumento da dose. Isto pode dever-se à grande variação de concentração plasmática que se obtém com a mesma dose oral em indivíduos com diferentes taxas de absorção e metabolismo dos antidepressivos.

\section{Recaída após a interrupção}

Os estudos que avaliam as taxas de recaída do TOC após a suspensão do tratamento são poucos e feitos com amostras pequenas, mas todos demonstram que o retorno dos sintomas é regra, e não exceção. Thoren et al, ${ }^{21} \mathrm{em}$ um estudo retrospectivo com 15 pacientes tratados com clomipramina, observaram que seis deles recaíram em poucas semanas após a interrupção do tratamento. A substituição da clomipramina pelo placebo acarretou, em 7 semanas, a recaída de $89 \%$ dos pacientes estáveis com clomipramina por até 27 meses. ${ }^{22}$ Um estudo duplo-cego com crianças e adolescentes que responderam à clomipramina observou que oito em nove recaíram quando ela foi substituída pela desipramina por dois meses. Apenas dois dos 11 que se mantiveram em uso da clomipramina recaíram no mesmo período. ${ }^{23}$ Pato et $\mathrm{al}^{24}$ observaram que quatro em cinco pacientes $(80 \%)$ recaíram em até 12 semanas após a retirada da fluoxetina. $\mathrm{O}$ tempo maior para a recaída pode ser devido à meia vida mais longa da fluoxetina e de seu metabólito ativo, a norfluoxetina.

\section{Manutenção}

Há poucos estudos para orientar o clínico no tratamento farmacológico em longo prazo do TOC, ${ }^{25}$ mas já é possível afirmar que pacientes que mantêm o tratamento medicamentoso recaem menos do que aqueles que o interrompem. Dois estudos duplocegos controlados com placebo demonstraram a superioridade da clomipramina ${ }^{2}$ e da sertralina ${ }^{5}$ nos pacientes tratados durante um ano. A melhora máxima com a sertralina ocorreu na $20^{\mathrm{a}}$ semana de tratamento e se manteve na maioria dos pacientes que continuaram a utilizá-la por mais um ano de forma aberta. ${ }^{26}$

Ao contrário da depressão, em que o tratamento de manutenção deve ser feito com as mesmas doses utilizadas na fase aguda, as doses de manutenção para o TOC ainda não estão definidas. Pato et $\mathrm{al}^{27}$ observaram que pacientes estabilizados por cerca de 10 meses com a clomipramina toleraram, sem piorar, uma redução de $40 \%$ das doses iniciais. Mundo et $\mathrm{al}^{28}$ relataram que a redução de até $66 \%$ das doses de clomipramina e fluvoxamina utilizadas na fase aguda não resultaram em taxas mais elevadas de recaída em 30 pacientes acompanhados por 102 dias. Porém, num estudo recente com pacientes com TOC tratados por um ano, ${ }^{29}$ a fluoxetina só foi superior ao placebo na prevenção de recaídas na dose de $60 \mathrm{mg} /$ dia. Pacientes utilizando $40 \mathrm{mg} /$ dia e $20 \mathrm{mg} /$ dia recaíram tanto quanto os que utilizaram placebo. Enquanto a questão da dose na fase de manutenção não estiver definida, recomenda-se manter as mesmas doses utilizadas na fase aguda, observada a tolerância do paciente aos efeitos colaterais.

Após a resposta, o tratamento farmacológico deve prosseguir por no mínimo 1 a 2 anos. ${ }^{11}$ A redução não deve ser feita com rapidez superior a $25 \%$ da dose a cada dois meses. Pacientes que mantêm sintomatologia clinicamente relevante, apesar do tratamento, são candidatos à potencialização com outros medicamentos ou com terapia cognitivo-com- 
portamental (TCC). A TCC diminui as taxas e a rapidez das recaídas após a parada da medicação e, sempre que disponível, deve ser utilizada desde o início do tratamento isoladamente ou em combinação com a psicofarmacoterapia. A TCC pode ser a primeira indicação nos casos leves (veja Wielenska et al neste número).

\section{Conclusão}

Está bem estabelecida a eficácia dos IRS (seletivos e não seletivos) no tratamento do TOC, o que os torna medicamentos de primeira escolha. Embora raramente os pacientes fiquem assintomáticos, a maioria deles obtém alívio significativo dos sintomas. Os ISRS produzem menos efeitos colaterais e são bem tolerados em doses altas; a clomipramina apresenta uma resposta mais robusta. Pacientes que não responderam a um IRS podem fazê-lo a outros. Uma vez que o curso do TOC tende a ser flutuante, com pioras e melhoras, o tratamento de manutenção está indicado na maioria dos casos.

\section{Referências}

1. Fernandez CE, Lopez-Ibor-Aliño J. La monoclorimipramina en enfermos psiquiatricos resistentes a otros tratamientos. Actas Luso-Españolas de Neurologia y Psiquiatria 1967;26:119-47.

2. DeVeaugh-Geiss J, Katz R, Landau P, Summit NJ, Akiskal H, Ananth J, et al. Clomipramine in the treatment of patients with obsessive-compulsive disorder: the clomipramine collaborative study group. Arch Gen Psychiatry 1991;48:730-8.

3. Tollefson GD, Rampey AH, Potvin JH, Jenike MA, Rush AJ, Dominguez RA, et al. A multicenter investigation of fixed-dose fluoxetine in the treatment of obsessive-compulsive disorder. Arch Gen Psychiatry 1994;51:559-67.

4. Goodman WK, Kozak MJ, Liebowitz M, White KL. Treatment of obsessive-compulsive disorder with fluvoxamine: a multicenter, double-blind, placebo controlled trial. Int Clin Psychopharmacol 1996;11:21-9.

5. Greist JH, Chouinard G, Duboff E. Double-blind parallel comparison of three dosages of sertraline and placebo in outpatients with obsessive-compulsive disorder. Arch Gen Psychiatry 1995;52:289-95.

6. Zohar J, Judge R. Paroxetine versus clomipramine in the treatment of obsessive-compulsive disorder. Br J Psychiatry 1996;169:468-74.

7. Pato MT. Beyond depression: citalopram for obsessivecompulsive disorder. Int Clin Psychopharmacol 1999;14(suppl 2):19-26.

8. Rauch SL, O'Sullivan RL, Jenike MA. Open treatment of obsessive-compulsive disorder with venlafaxine: a series of ten cases. J Clin Psychopharmacol 1996;16:81-4.

9. Yaryura-Tobias JA, Neziroglu FA. Venlafaxine in obsessivecompulsive disorder. Arch Gen Psychiatry 1996;53:653-4.

10. Greist JH, Jefferson JW. Pharmacotherapy for obsessivecompulsive disorder. Br J Psychiatry 1998;173(suppl 35):64-70.

11. March JS, Frances A, Carpenter D, Kahn DA, editors. The Expert Consensus Guideline Series: treatment of obsessive-compulsive disorder. J Clin Psychiatry 1997;58(suppl 4):4-64.

12. Todorov C, Freeston MH, Borgeat F. On the pharmacotherapy of obsessive-compulsive disorder: is a consensus possible? Can J Psychiatry 2000;45:257-62.

13. Van Balkom AJ, Van Oppen P, Vermeulen AWA. A meta-analysis on the treatment of obsessive-compulsive disorder: a comparison of antidepressants, behavior, and cognitive therapy. Clin Psychol Review 1994;14:359-81.

14. Greist JH, Jefferson JW, Kobak KA. Efficacy and tolerability of serotonin transport inhibitors in obsessive-compulsive disorder: a meta-analysis. Arch Gen Psychiatry 1995;52:53-60.

15. Piccinelli M, Pini S, Bellantuono C. Efficacy of drug treatment in obsessive-compulsive disorder: a meta-analytic review. Br J Psychiatry $1995 ; 166: 424-43$.
16. Stein DJ, Spadaccini E, Hollander E. Meta-analysis of pharmacotherapy trials for obsessive-compulsive disorder. Int Clin Psychopharmacology 1995;10:11-8.

17. Abramowitz JS. Effectiveness of psychological and pharmacological treatments for obsessive-compulsive disorder: a quantitative review. J Cons Clin Psychiatry 1997;65:44-52.

18. Kobak KA, Greist JH, Jefferson JW. Behavioral versus pharmacological treatments of obsessive-compulsive disorder: a meta-analysis. Psychopharmacology 1998;136:205-16.

19. Fontenelle L, Nardi AE, Marques C, Figueira I, Possidente E, Versiani M. Inibidores seletivos da recaptação da serotonina no transtorno obsessivo-compulsivo. J Bras Psiquiatria 1998;46:487-98.

20. Pigott TA, Pato MT, Berstein SE, Grover GN, Hill JL, Tolliver TJ, et al. Controlled comparisons of clomipramine and fluoxetine in the treatment of obsessive-compulsive disorder: behavioral and biological results. Arch Gen Psychiatry 1990;47:926-32.

21. Thoren P, Asberg M, Cronholm B, Jornestedt L, Traskman L. Clomipramine treatment of obsessive-compulsive disorder I: a controlled clinical trial. Arch Gen Psychiatry 1980;37:1281-5.

22. Pato MT, Zohar-Kadouch R, Zohar J, Murphy DL. Return of symptoms after discontinuation of clomipramine in patients with obsessive-compulsive disorder. Am J Psychiatry 1988;145:1521-5.

23. Leonard HL, Swedo SE, Lenane MC, Rettew DC, Cheslow DL, Hamburger SD, et al. A double-blind desipramine substitution during long term clomipramine treatment in children and adolescents with obsessive-compulsive disorder. Arch Gen Psychiatry 1991;48:922-7.

24. Pato MT, Murphy DL, DeVane CL. Sustained plasma concentratios of fluoxetine and/or norfluoxetine four and eight weeks after fluoxetine discontinuation. J Clin Psychopharmacol 1991;11:224-5.

25. Montgomery AS. Long term management of obsessive-compulsive disorder. Int Clin Psychopharmacol 1996;11(suppl 5):23-9.

26. Rasmussen S, Hackett E, DuBoff E, Greist J, Halaris A, Koran LM, et al. A 2-year study of sertraline in the treatment of obsessive-compulsive disorder. Int Clin Psychopharmacol 1997;12:309-16.

27. Pato MT, Hill JL, Murphy DL. A clomipramine dosage reduction study in the course of long-term treatment of obsessivecompulsive disorder patients. Psychopharmacol Bull 1990;26:211-4.

28. Mundo E, Bareggi SR, Pirola R, Bellodi L, Smeraldi E. Long-term pharmacotherapy of obsessive-compulsive disorder: a doubleblind controlled study. J Clin Psychopharmacol 1997;17:4-10.

29. Romano S, Goodman W, Tamura R, Gonzales J. Long-term treatment of obsessive-compulsive disorder after an acute response: a comparison of fluoxetine versus placebo. J Clin Psychopharmacol 2001;21:46-52.

\section{Correspondência: Carla Marques}

Av. Princesa Isabel, 150/401 Copacabana -22010-011 Rio de Janeiro, RJ

Tel.: (0xx21) 294-8429/542-3694 -E-mail: csms@unysis.com.br 